\title{
Comparison of Structural Modification of Sugarcane Bagasse Cellulose Regenerated from Three Imidazolium-Based Ionic Liquids

\author{
Lihong Cui ${ }^{1, a^{*}}$, Xiaoyi Wei ${ }^{1, b}$, Tiaokun Fu ${ }^{1, c}$, Gang Chang ${ }^{1, d}$, Bing Tang ${ }^{3, e}$ and \\ Qin Feng ${ }^{2, f}$
}
1 Agriculture Products Processing Research Institute, Chinese Academy of Tropical Agricultural Sciences, Zhanjiang 524001, China

2 South Subtropical Crops Research Institute, Chinese Academy of Tropical Agricultural Sciences, Zhanjiang 524001, China
${ }^{3}$ Spice and Beverage Research Institute, Chinese Academy of Tropical Agricultural Sciences, Wanning 571533, China

alh_cui@126.com, ${ }^{b}$ weixywei@163.com, loveflyfree@126.com, ${ }^{d}$ changgang86@126.com, etangb@catas.cn, fengqin@163.com

\section{Keywords: Sugarcane bagasse; Cellulose; Ionic liquids; Comparison; Structure}

Abstract. Sugarcane bagasse cellulose (SBC) was dissolved in three imidazolium-based ionic liquids (ILs) $\left[\right.$ Emim]DEP, $[$ Emim $] \mathrm{Ac}$ and $[\mathrm{Bmim}] \mathrm{Cl}$ at the same conditions $\left(1 \%\right.$ mass loading, $\left.110^{\circ} \mathrm{C}\right)$. Among the three ILs, [Emim]DEP has the highest dissolution rate and regeneration yield of SBC. Both the original and regenerated SBC samples were characterized by X-ray diffraction, infrared spectroscopy, scanning electron microscopy and thermogravimetric analysis. The results showed that the crystal structure of SBC was rearranged from cellulose I to cellulose II after regeneration from all the three ILs, but different IL leads to a different degree of crystallinity index (CrI) reduction. The $\mathrm{CrI}$ of $\mathrm{SBC}$ regenerated from [Bmim]Cl was significantly decreased from $73.35 \%$ to $25.08 \%$, which is the lowest in all samples. Surprisingly, the CrI value of regenerated SBC from [Emim]DEP was just a little higher than that of [Bmim] Cl, although the dissolution time of [Emim] DEP is much shorter than that of $[\mathrm{Bmim}] \mathrm{Cl}$. It was also found that the regenerated SBC from [Emim]DEP had better thermal stability when compared with the untreated and regenerated SBC from the other two ILs.

\section{Introduction}

Cellulose, the major skeletal component of plant biomass, presents an abundant renewable and biodegradable organic polymer on the earth. Along with the decreasing reserves of fossil fuel resources, cellulose has been proposed as sustainable alternatives to petroleum-based feedstock. Due to its numerous inter- and intra-molecular hydrogen bonds and high crystallinity, cellulose is chemically stable and insoluble in most conventional solvents [1]. Therefore, it is very important to find effective pretreatment method to break the hydrogen bond network structure of cellulose. Ionic liquids (ILs) receive significant and continuously growing interest lately as a new type of green solvent for lignocellulosic biomass [2]. Decrystalisation of cellulose is a known and unique effect of ILs pretreatment [3]. Among process parameters for cellulose dissolving in ILs, the solution temperature has a significant influence on solution time and performance of regenerated cellulose[4]. But as far as we know, there is little report about the influence of different ILs on the dissolution time of cellulose and performance of regenerated cellulose at the same dissolution temperature.

In this study, three different imidazolium-based ILs [Emim]DEP, [Emim]Ac and [Bmim]Cl were used to dissolve sugarcane bagasse under microwave radiation at the same temperature. The dissolution time and regeneration yield of cellulose for each ILs was compared. The effect of these ILs treatment on the structural modification has also been fully investigated. The structure of original and regenerated cellulose was analyzed by Fourier transformed infrared spectrophotometry (FT-IR), X-ray diffraction (XRD), scanning electron microscopy (SEM) and thermogravimetric analysis (TG). 


\section{Experimental Section}

Materials. Sugarcane bagasse was obtained from a local sugar factory (Zhanjiang, China). The ionic liquids $[\mathrm{Bmim}] \mathrm{Cl},[\mathrm{Emim}] \mathrm{Ac}$ and [Emim]DEP were synthesized as described in previous literature [5-7], and their structure were verifed by FT-IR and ${ }^{1} \mathrm{H}$ NMR. All other reagents and solvents were of analytical grade.

Extraction of sugarcane bagasse cellulose. Sugarcane bagasse was milled to particles passing through a $250 \mu \mathrm{m}$ sieve. To extract cellulose from this agricultural waste, $3 \mathrm{wt} \%$ nitric acid and 1.5 wt \% sodium hydrate were employed sequentially based on the method that reported elsewhere [8]. The sugarcane bagasse cellulose (SBC) was dried in vacuum freeze drying equipment and then stored until used.

Dissolution and regeneration of SBC. Dried SBC power at a mass loading of $1 \%$ was soaked in $[\mathrm{Bmim}] \mathrm{Cl},[\mathrm{Emim}] \mathrm{Ac}$ and $[\mathrm{Emim}] \mathrm{DEP}$ respectively. Then the mixture was continuously stirred in the microwave oven (Qpro-M, Questron Inc., Canada) at $110{ }^{\circ} \mathrm{C}$.The dissolution process of SBC in ILs was observed by polarizing microscope. When the cellulose was completely dissolved, the solution was stopped heating and the dissolution time was calculated. After cooling down to room temperature, cellulose was regenerated from the solution by the addition of distilled water. The recovered solid was washed with deionized water to remove excess ILs. Finally, the regenerated cellulose was centrifuged and lyophilized until reaching a constant weight. The yield of regenerated SBC was calculated as follows:

$$
\text { Yield }(\%)=\frac{\mathrm{m}_{1}}{\mathrm{~m}_{0}} \times 100
$$

where $\mathrm{m}_{0}$ is the dry-weight of SBC before dissolved, $\mathrm{m}_{1}$ is the dry-weight of regenerated SBC.

Characterization. Original and regenerated SBC samples were analyzed with XRD (X'Pert, Philip, Germany) for crystal structure, FT-IR (Spectrum GX-1, PerkinElmer, USA) for chemical structure, SEM (S-4800, HITACHI, Japan) for surface morphology. In addition, thermal stability was analyzed with a Synchronous Thermal Analysis (STA449C/4/G, Netzsch, Germany).

\section{Results and Discussion}

Dissolution and regeneration of SBC. The dissolution of SBC sample in ILs [Bmim]Cl, [Emim]Ac and [Emim]DEP was investigated under the same conditions $\left(1 \%\right.$ mass loading, $\left.110^{\circ} \mathrm{C}\right)$. Table 1 listed the dissolution time and regeneration yields of SBC with different ILs pretreatment. The shortest time was obtained in [Emim]DEP, which was only $25 \mathrm{~min}$. It took a little longer time for complete dissolution of SBC in [Emim]Ac. While the time for SBC dissolving in [Bmim]Cl was up to $288 \mathrm{~min}$. But the yield of regenerated SBC was greatly reduced as the dissolution time. In the process of dissolution, cellulose solubilized in the ILs may also undergo depolymerization into low molecular weight water-soluble components [2]. These water-soluble components were washed away during the washing step, which resulting in loss of regenerated cellulose quality. Based on these points, the higher regeneration yield of SBC in [Emim]DEP was due to reduce the depolymerization of cellulose.

$\underline{\text { Table } 1 \text { Dissolution time and regeneration yield of SBC with different ILs }}$

\begin{tabular}{ccc}
\hline IL & $\begin{array}{c}\text { Dissolution time } \\
{[\mathrm{min}]}\end{array}$ & $\begin{array}{c}\text { Yield } \\
{[\%]}\end{array}$ \\
\hline$[$ Bmim $] \mathrm{Cl}$ & 288 & 60.35 \\
{$[$ Emim]Ac } & 44 & 87.31 \\
{$[$ Emim $][\mathrm{DEP}]$} & 25 & 90.78 \\
\hline
\end{tabular}

XRD analysis. XRD patterns of original and regenerated SBC from each ILs were shown in Fig.1. The diffractogram of original SBC sample exhibits diffraction typical of cellulose I with diffraction peaks at $16^{\circ}$ and $22^{\circ}$. However, the diffraction curves of regenerated SBC samples from each ILs 
were the typical diffraction patterns of cellulose II by the presence of the broad crystalline peak at around $12.5^{\circ}$ and $20.0^{\circ}$ [9]. The results indicated that the crystal structure of SBC was rearranged from cellulose I to cellulose II during the solubilization and regeneration process. The crystallinity index (CrI) of original and regenerated SBC were estimated by using the empirical method of Segal [10], and the results are summarized in Table 2. It was observed that the CrI of the solids recovered from all three ILs treatments were significantly lower than that of the untreated SBC. Moreover, different IL leads to a different degree of CrI reduction. Regenerated SBC from [Bmim]Cl have the lowest CrI value of $25.08 \%$ in all the samples. It may be caused by the longest time of SBC dissolution in [Bmim]Cl. Surprisingly, the CrI value of SBC treated by [Emim]DEP was lower than that treated by [Emim]Ac. This result may demonstrate that the reduction of cellulose CrI is not related to the dissolution time of cellulose in ILs.

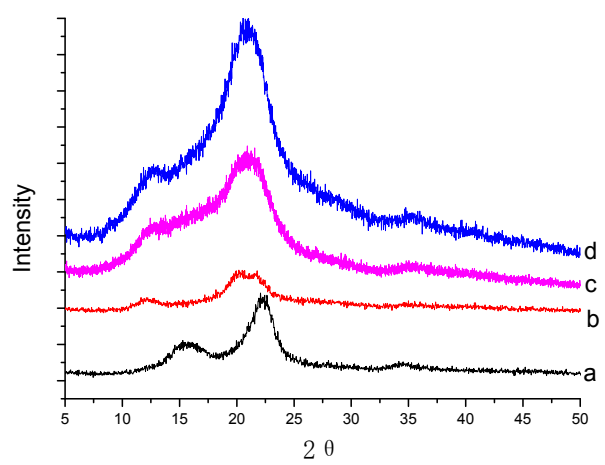

Fig.1 XRD patterns of original (a) and regenerated SBC from [Bmim]Cl (b), [Emim]Ac (c) and $[\operatorname{Emim}] \mathrm{DEP}(\mathrm{d})$

Table 2 CrI of original and regenerated SBC

\begin{tabular}{cc}
\hline Sample & CrI [\%] \\
\hline Original SBC & 73.35 \\
Regenerated SBC from [Bmim]Cl & 25.08 \\
Regenerated SBC from [Emim]Ac & 38.26 \\
Regenerated SBC from [Emim]DEP & 31.48 \\
\hline
\end{tabular}

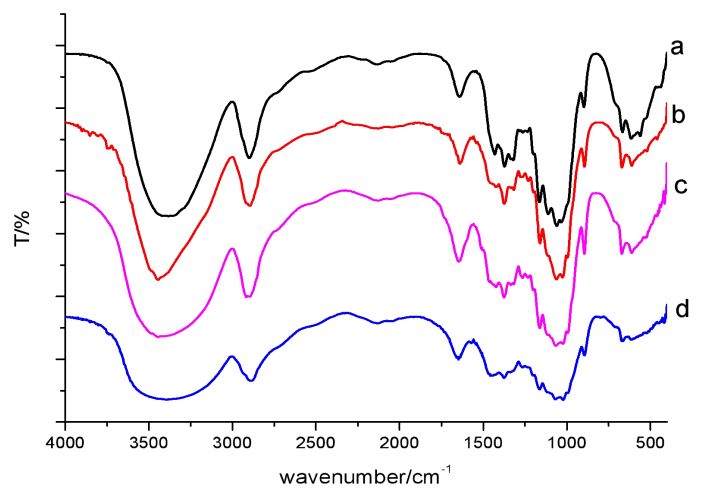

Fig.2 FT-IR spectra of original (a) and regenerated SBC from [Bmim]Cl (b), [Emim]Ac (c) and $[\operatorname{Emim}] \mathrm{DEP}(\mathrm{d})$

FT-IR Spectra. Fig.2 illustrates the FT-IR spectra of original and regenerated SBC from each ILs. The peak associated with hydrogen bonds is $3379 \mathrm{~cm}^{-1}$ for original SBC, while the peak for 
regenerated SBC moved to the red shift. It suggested that the inter- and intra- molecular hydrogen bonds between cellulose chains became weaker after ILs treatment [11]. The peak at $1432 \mathrm{~cm}^{-1}$ in original SBC is assigned to the intra-molecular hydrogen bonds [12]. The peak for regenerated SBC was also shifted to a higher wavenumber. It indicated that the number of intra-molecular hydrogen bonds in ILs-treated SBC decreased. These results indicated a change in the crystal structure of SBC regenerated from ILs, which was in accordance with the conclusion derived from XRD analysis.

Surface Morphology Observation. SEM was a useful tool to investigate the surface structure of lignocellulosic biomass. Fig.3a show that the surface structure of original SBC was tightness and smooth. According to the SEM images (Fig.3b-d), the solubilization and regeneration of SBC in ILs lead to a relatively more porous and incompact network of cellulose. This morphological structure could increase the contact area of cellulose with active substances and improve the reactivity of cellulose. When comparing the surface structure of SBC treated by three ILs, it was obviously seen that $[\mathrm{Bmim}] \mathrm{Cl}$ - treated sample was the most rough and porous. This result is in accordance with the reduction of SBC CrI after treated by three ILs.
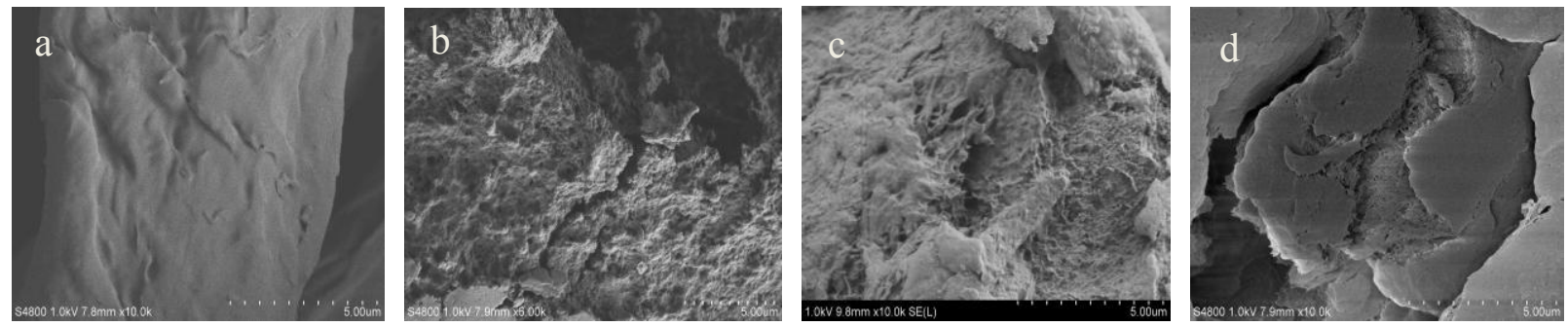

Fig.3 SEM images of original (a) and regenerated SBC from [Bmim]Cl (b), [Emim]Ac (c) and $[\operatorname{Emim}] \mathrm{DEP}(\mathrm{d})$

Thermal analysis. Fig. 4 displayed the TGA and DTG curves of original and regenerated SBC. The original SBC started to decompose at $331.7^{\circ} \mathrm{C}$, whereas the regenerated SBC from [Bmim]Cl, [Emim]Ac and [Emim]DEP began to decompose at $270.5{ }^{\circ} \mathrm{C}, 270.5^{\circ} \mathrm{C}$ and $296.5{ }^{\circ} \mathrm{C}$ respectively. These figures implied that the thermal stability of regenerated SBC were lower than that of original cellulose. In addition, the residual of [Emim]DEP-treated SBC was higher than the other samples. So, thermal stability of SBC treated by [Emim]DEP was much better than that treated by [Emim]Ac and $[\mathrm{Bmim}] \mathrm{Cl}$.
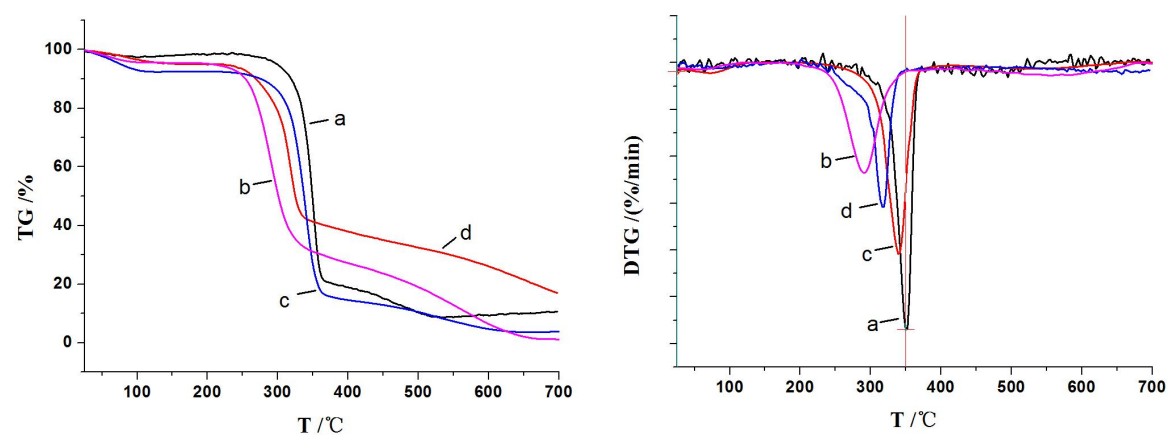

Fig.4 TGA and DTG spectra of original (a) and regenerated SBC from [Bmim]Cl (b), [Emim]Ac (c) and $[\operatorname{Emim}] \mathrm{DEP}(\mathrm{d})$

\section{Conclusions}

As the dissolution time for SBC in ionic liquid increased, the yield of regenerated SBC was greatly reduced. Among the three ionic liquids, [Emim]DEP has the highest dissolution rate and regeneration yield of SBC. According to the structural comparison of the regenerated cellulose from different ionic liquid, the CrI value of SBC treated by [Emim]DEP was just a little higher than that of [Bmim] Cl, and lower than that of [Emim]AC-treated sample. In addition, the regenerated SBC from [Emim]DEP had better thermal stability than untreated substrate and regenerated SBC from the other two ILs. 


\section{Acknowledgments}

The authors are extremely grateful for the financial support from National Nonprofit Institute Research Grant of CATAS (No. 1630062013012 \& No.1630062015016) and Zhanjiang agricultural research project (No. 2016A03019).

\section{References}

[1] B. Hinterstoisser, M. Akerholm and L. Salmén: Biomacromolecules, Vol.4 (2003), p.1232-1237

[2] E. Bahcegul, S. Apaydin and N. I. Haykir: Green Chemistry, Vol.14 (2012), p.1896-1903

[3] S.K. Karatzos, L.A. Edye and W.O.S. Doherty: Biotechnology for Biofuels, Vol.5 (2012), p.62

[4] D. Zhao, H. Li and J. Zhang: Carbohydrate Polymers, Vol.87 (2012), p.1490-1494

[5] Jihua Li, Xiaoyi Wei and Qinghuang Wang: Carbohydrate Polymers, 2012,Vol.90 (2012), p.1609-1613.

[6] Meng Wang, Lihong Cui and Jihua Li: Study on Dissolution of Cellulose in Ionic Liquid 1-Ethyl-3-Methylimidazolium Acetate[J]. New Chemical Materials, Vol.42 (2014), p.122-124 (in Chinese)

[7] E. Kuhlmann, S. Himmler and H. Giebelhaus: Green Chemistry, Vol.9 (2007), p.233-242

[8] D. H. Han, X.Y. Wei and J.H. Li: Chinese Agricultural Science Bulletin, Vol.14 (2013), p.113-117 (in Chinese)

[9] Y. Cao, H.M. Tan: Enzyme and Microbial Technology, Vol.36 (2005), p.314-317

[10] L. Segal , L. Creely and A.E. Martin: Textile Research Journal, Vol.29 (1959), p.786-794

[11] A. Watanabe, S.Morita and Y.Ozaki: Biomacromolecules, Vol.8 (2007), p.2969-2975

[12] H. Zhang, J. Wu and Zhang J: Macromolecules, Vol.38 (2005), p.8272-8277 